Revista de

Contabilidade e

Organizações

www.rco.usp.br
DOI: http://dx.doi.org/10.11606/rco.v7i19.55424
Journal of

Accounting and

Organizations

\title{
Inovação de práticas de controle gerencial e fontes de informação
}

José Carlos T. Oyadomaria ; Andson B. de Aguiar ${ }^{\mathrm{b}}$; Yen-Tsang Chen ${ }^{\mathrm{c}}$; Ronaldo G. Dultra-de-Lima ${ }^{\mathrm{a}}$

${ }^{a}$ Universidade Presbiteriana Mackenzie.

${ }^{b}$ Faculdade de Economia, Administração e Contabilidade - USP-Universidade de São Paulo.

c Escola de Administração de Empresas - Fundação Getúlio Vargas.

\section{Informações do Artigo}

Histórico do Artigo

Recebido: 8 de maio de 2012

Aceito: 31 de outubro de 2013

Palavras chave:

Inovação

Práticas de controle gerencial

Fontes de conhecimento

Mudanças.

\section{Resumo}

Este estudo avaliou as associações entre a forma como os profissionais de controladoria obtém obtêm informações e os perfis de mudanças de práticas de controle gerencial, um assunto ainda pouco explorado (STONE et al., 2000). Com base em 80 respostas de profissionais das áreas de controladoria, contabilidade e finanças de empresas brasileiras, e com o uso da técnica de análise de clusters, identificaram-se quatro diferentes perfis de inovação de práticas de controle gerencial denominados "Não Inovadores", "Inovadores Frequentes", "Inovadores Incrementais" e "Inovadores Radicais". Os principais resultados, demonstraram que: (1) a fonte interna de informação é a mais importante, (2) não há diferença na forma como se obtém a informação e os diferentes perfis de mudanças de práticas de controle gerencial, indicando que outros fatores podem explicar os diferentes perfis de mudanças de práticas de controle gerencial. A única exceção se refere ao agrupamento denominado "Não Inovadores", em que as fontes de informação como jornais e revistas de negócios, artigos acadêmicos e associações de classe são diferentes em relação aos demais perfis, com médias maiores, o que pode indicar uma postura crítica em relação às inovações ou mesmo que estas essas organizações estão ainda buscando informações para um futuro movimento de mudanças.

Copyright $(2013$ FEA-RP/USP. Todos os direitos reservados

\section{INTRODUÇÃO}

A inovação abrange mais que o processo de renovação de produtos e serviços, ela inclui os processos operacionais para produzir, vender e entregar esses produtos e serviços, e também os processos organizacionais, bem como novas formas de desenho organizacional ou de gerenciamento (SUNDBO, 2001). Essas inovações mais relacionadas às técnicas de gestão e estruturas organizacionais têm sido estudadas sob o nome de inovações organizacionais (PETTIGREW; MASSINI, 2003) e são caracterizadas pelo processo de mudança, o qual a literatura intitulou de change management.

Adefinição da literatura é que inovação organizacional é algo novo, não necessariamente novo enquanto campo de conhecimento, mas sim sob a perspectiva da organização, isto é, inovação é algo que a organização ainda não possuía, e este é o conceito adotado pelos especialistas no tema (MOL; BIRKINSHAW, 2009). Os principais vetores das inovações organizacionais na perspectiva da literatura baseada na prática apontam para a ampliação

Autor Correspondente: Tel +55 11 2114-8273

E-mail : J. C. T. Oyadomari (oyadomari@mackenzie.br); A. B. Aguiar (abraga@usp.br); Y. Chen (yentsang@gvmail.br); R. G. Dultra-de-Lima (ronaldo.lima@gvmail.br)

Universidade Presbiteriana Mackenzie. R. da Consolação 896 - Consolação 01302-907 - São Paulo, SP - Brasil das fronteiras organizacionais, com destaque para as redes de empresas, a tendência de estilos gerenciais mais cooperativos e o foco em uma organização criativa e aprendizado (PETTIGREW; MASSINI, 2003).

Portanto, na perspectiva contingencial seria esperado que essas inovações organizacionais produzissem mudanças nas práticas de controle gerencial, uma vez que o sistema de controle gerencial deveria se ajustar a essas novas formas de estrutura organizacional e estilos de gestão, conforme propuseram Chenhall (2008) e Berry et al. (2009). Embora haja um forte clamor das pesquisas apontando para a baixa aderência, na prática, das chamadas técnicas contemporâneas de controle gerencial, esses estudos têm optado por identificar a adoção ou não adoção de um conjunto de práticas de controle gerencial, geralmente classificando-as em tradicionais e contemporâneas, em vez de identificar os movimentos de mudança de práticas de controle gerencial.

Identificar esses movimentos de mudanças e práticas de controle gerencial é importante, pois pode levantar tendências indicando os movimentos atuais e futuros das organizações em relação às práticas de controle gerencial. Nesse sentido, Sulaiman e Mitchell (2005) desenvolveram uma tipologia para classificar os tipos de mudança de práticas de contabilidade gerencial, classificando-as em: Adição, Reposição, Modificação Operacional, Modificação de Relatório e Remoção. Entende-se que essa tipologia é adequada para identificar 
os perfis de mudança de práticas de controle gerencial, pois de alguma forma esses estágios podem ser associados à tipologia de inovações classificada como radical ou incremental (DAVILA et al., 2009), além de identificar comportamentos mais direcionados às melhorias contínuas ou mesmo abandono de práticas.

O processo de inovação, que é na realidade um processo de mudança organizacional, é bastante influenciado pelo processo de gestão de conhecimento, uma vez que a inovação é resultante da combinação de conhecimento existente com novos conhecimentos (ALMEIDA et al., 2003). E é pelo compartilhamento de informações entre os membros que o conhecimento se institucionaliza por meio de diversos artefatos (FLEURY; FLEURY, 2000). Portanto, embora o conhecimento tenha origem na mente de cada indivíduo, nas organizações ele se encontra em rotinas, processos, práticas e normas organizacionais (VASCONCELOS, 2007), e é através do compartilhamento de informações entre os membros que o conhecimento se institucionaliza por meio de diversos artefatos (FLEURY; FLEURY, 2000).

$\mathrm{O}$ processo de geração de conhecimento passa pela obtenção de informação (CHENHALL, 2005), e quanto maior o grau de obtenção de informação utilizada pela empresa, maior é o grau de introdução de novas práticas gerenciais, uma vez que os gestores buscam nas fontes externas e internas o conhecimento necessário para aperfeiçoar as práticas gerenciais tais como seminários, consultorias, rede de contato, livros, dentre outros (MOL; BIRKINSHAW, 2009).

A situação-problema que se configura é se de um lado os gestores obtêm informações de diferentes fontes, consoante às afirmações de Mol e Birkinshaw (2009), de outro lado, existe um perfil de inovação de práticas de controle gerencial conforme a tipologia desenvolvida por Sulaiman e Mitchel (2005). Ainda são poucos os estudos sobre como os profissionais de controladoria adquirem informações e as transformam em inovações (STONE et al., 2000), constituindo-se este fenômeno como uma oportunidade de pesquisa.

Dessa forma, o presente estudo se propõe a responder a seguinte questão de pesquisa: os perfis de mudanças de práticas de contabilidade gerencial são diferentes em função da forma como os profissionais de controladoria obtêm informação?

O objetivo geral desta pesquisa é identificar se existem associações em termos de fontes de obtenção de informação e perfis de mudança de práticas de controle gerencial. Como objetivos específicos, este estudo se propõe a identificar:

- As fontes de obtenção de informação mais importantes; e

- Os perfis de mudanças de práticas de controle gerencial.

Para fins desta pesquisa as duas variáveis são conceituadas da seguinte forma:

- Fontes de obtenção de informação são definidas como sendo os recursos utilizados pelos membros da organização para obtenção de conhecimento. Geralmente, são classificadas como fontes internas e externas, conforme Mol e Birkinshaw (2009);

- Perfis de mudança de práticas de controle gerencial são definidos como as formas de mudança desenvolvidas nos processos de controle gerencial, que podem ser inovações, melhorias incrementais e mesmo estabilidade.

Os resultados deste estudo podem ajudar a entender em quais fontes os profissionais de controladoria buscam informações e conhecimento, permitindo que desenvolvedores de inovações em controle gerencial possam utilizar esses canais para divulgação, obtendo maiores chances de institucionalização dessa inovação.

Espera-se que os resultados ajudem a identificar os movimentos de mudança de práticas de controle gerencial nas empresas brasileiras e a mostrar que existem diferentes grupos de empresas, além de poder identificar associações entre os perfis de inovação e a forma como estes gestores obtêm as informações.

Entende-se que esses resultados são úteis para a literatura ao fornecer não somente o grau de adoção, mas principalmente quais tipos de informações podem estar associados com a adoção ou a não adoção de técnicas de controle gerencial.

\section{REFERENCIAL TEÓRICO}

\subsection{Informação e conhecimento}

Consoante à questão de pesquisa, o referencial teórico abrangerá os construtos: Fontes de informação e perfis de mudança de práticas de controle gerencial.

No campo da estratégia, o conhecimento é considerado como fonte de vantagem competitiva e pode ajudar a explicar a diferença de desempenho das empresas (GRANT, 1996). Por outro lado, com os avanços da tecnologia, o conhecimento também pode se tornar obsoleto mais rapidamente; portanto, é fundamental identificar como a organização obtém novas informações para gerar novos conhecimentos.

Na visão de Nonaka et al. (2001), há uma diferença entre informação e conhecimento. Para eles, a informação é um fluxo de mensagens, enquanto que o conhecimento é criado pelos diversos fluxos de informação. Já Cho (2006) prefere discutir que conhecimento e informação são resultantes da ação humana que combina signos, sinais e artefatos em espaços sociais e físicos e, portanto, é por meio do uso, prática e reflexões que a informação se transforma em conhecimento. Esse conhecimento provê uma contribuição à inovação nas empresas e por meio da gestão desse conhecimento, estas podem transformálo em inovações administrativas e técnicas (CHEN; HUANG, 2009).

A despeito da discussão de como informação se torna conhecimento, e ainda baseado na questão de pesquisa, o foco deste trabalho é identificar uma parte do processo de gestão de conhecimento, geralmente dividido em aquisição, compartilhamento e aplicação (CHENHALL, 2005), que é a etapa de aquisição de informações. Nesse sentido, o foco do estudo é a aquisição de informações.

\subsection{Fontes de obtenção de informação}

Uma tipologia adotada é a que classifica as fontes de obtenção de informações em internas, de mercado e profissionais. Mol e Birkinshaw (2009) utilizaram e detalharam essas categorias em:

1. Internas: dentro da empresa; e outras empresas do mesmo grupo.

2. Mercado: fornecedores de equipamento, materiais, componentes ou softwares; clientes ou consumidores; concorrentes; consultores; laboratórios comerciais/empresas de pesquisa e desenvolvimento.

3. Profissionais: conferências e reuniões 
profissionais; associações comerciais; revistas técnicas, bases de dados; e feiras e exposições.

Em linhas gerais, Mol e Birkinshaw (2009) argumentam que, quanto maior as fontes de conhecimento utilizadas pela empresa, maior é o grau de introdução de novas práticas gerenciais. Os autores utilizaram a variável "tamanho" como proxy de inovação, a qual é suportada pela teoria da contingência, em que quanto maior o tamanho da empresa, maior é a capacidade de obtenção de conhecimento, uma vez que a empresa tem acesso mais facilitado a contratação de consultores, treinamento, além de estar constantemente em contato com fornecedores, universidades e detentores de pesquisa e desenvolvimento.

Muito embora a tipologia desenvolvida por $\mathrm{Mol}$ e Birkinshaw (2009) seja importante, entende-se que ela foi desenvolvida para inovações organizacionais de forma abrangente, sendo necessário introduzir alguns itens mais aplicáveis ao departamento de Controladoria. Três variáveis foram incluídas tendo esse objetivo como pano de fundo:

- Livros - os livros têm sido apresentados como importantes no papel da institucionalização (SCAPENS, 1994);

- Artigos acadêmicos - muito embora Van Helden et al. (2010), em um estudo com consultores e pesquisadores, tenham identificado que os consultores confiam principalmente em obter conhecimento por meio de contatos com colegas e em revistas profissionais, os pesquisadores preferem utilizar periódicos acadêmicos internacionais. Mesmo assim, nota-se uma busca por conhecimento fundamentado em pesquisas, já que consultores que atuam em empresas internacionais têm acesso à base de conhecimento disponibilizada pelos centros de pesquisas das consultorias. Além disso, essa variável foi incluída na tentativa de aferir a relevância da produção acadêmica para a prática (MALMI; GRANDLUND, 2009, BALDVINSDOTTIR et al., 2010);

- Rede de contatos - Calhou e Starbuck (2003) fornecem uma explicação dos relacionamentos entre informação e conhecimento. Para eles, as pessoas e as organizações adquirem informação por meio de redes sociais, e uma porção dessa informação se torna conhecimento (CALHOU; STARBUCK, 2003). Pettigrew e Massini (2003) também apontam o papel do compartilhamento de informação além das fronteiras organizacionais, como forma de gerar inovações organizacionais.

\subsection{Perfis de mudança de práticas de controle gerencial}

Existem duas abordagens do que pode ser caracterizada como inovação em práticas gerenciais: ela pode ser uma nova técnica no estado da arte do campo de conhecimento, ou ainda ser nova para uma organização específica e também não se restringir às técnicas propriamente ditas, mas também a mudança na forma de atuação gerencial (EMSLEY, 2005). Como o foco deste estudo não é identificar quais práticas estão sendo adotadas pelas empresas brasileiras, mas sim os diferentes perfis de mudança, é mais apropriado utilizar a tipologia proposta por Sulaiman e Mitchell (2005, p. 426), consoante o Quadro 1.

Após análise da tipologia de Sulaiman e Mitchell (2005), entendeu-se que havia uma lacuna que indicasse o comportamento de respondentes que não fizeram nenhuma mudança no período, indicando um elevado grau de estabilidade das práticas. $\mathrm{O}$ estudo da estabilidade, além de ser analisado em ensaios como de Aguiar e Guerreiro (2008), pode ser útil para entender o comportamento desses profissionais, principalmente, em uma época de adoção de novas normas contábeis e sistema digital de escrituração fiscal.

Quadro 1. Tipologia de mudanças em práticas de controle gerencial

1. Adição - introducão de novas técnicas como extensão do sistema de contabilidade gerencial, por exemplo, custo da qualidade, indicadores não monetários.

2. Reposição - introdução de novas técnicas como reposição de partes de técnicas já existentes, por exemplo, orçamento flexível com orçamento fixo, custeio por atividades dentro do sistema de custos.

3. Modificação do relatório - modificação da informação relatada, por exemplo, mudança de mensal para semanal dos relatórios de análise das variações orçamentárias, ou forma de apresentação de numérico para gráfica.

4. Modificação operacional - modificação de uma operação técnica do sistema de contabilidade gerencial, por exemplo, o uso de taxa predeterminada em oposição a uma taxa real, ou o uso de análise de regressão em vez de análise separando custos fixos e variáveis.

5. Redução - remoção de uma técnica de contabilidade gerencial sem reposição por outra, por exemplo, abandono do orçamento ou da análise do ponto de equilíbrio.

Fonte: adaptado de Sulaiman e Mitchell (2005)

Diante disso, um sexto tipo de mudança de práticas de controle gerencial seria a própria ausência de mudança representada pela preferência por uma estabilidade das práticas atualmente existentes na controladoria.

É de se esperar que o profissional que busca mais informações tenha uma propensão para gerar novas práticas de controle gerencial. De fato, Mol e Birkinshaw (2009) assumem a existência de um relacionamento entre fontes de conhecimento utilizadas pela empresa e grau de introdução de novas práticas gerenciais.

Dessa forma, baseado em Mol e Birkinshaw (2009), fundamenta-se a Hipótese H1: O perfil de mudança de práticas de contabilidade gerencial é diferente para profissionais que obtêm informação de diferentes fontes.

\section{PROCEDIMENTOS METODOLÓGICOS}

Classifica-se esta pesquisa como exploratória, uma vez que os estudos ainda podem ser considerados em estágios iniciais (FLICK, 2009), como descritiva, pois pretende descrever o que acontece em relação a como os profissionais obtêm informações, e como método quantitativo de análise (HAIR JR et al., 2009), uma vez que utilizou a técnica análise de agrupamentos e ANOVA para identificar associações entre fontes de informação e perfis de inovação de práticas de controle gerencial.

\subsection{Questionário e amostra}

Com o objetivo de garantir melhor entendimento das questões pelos respondentes, foi feito um pré-teste (DILLMAN, 2000) junto a um diretor financeiro de uma 
indústria de autopeças e dois professores doutores com experiência em pesquisas do tipo survey.

A população considerada foi a de gerentes de controladoria, gerentes financeiros e gerentes de contabilidade de empresas brasileiras de médio e grande porte. Porém, dado que não existe uma base unificada de empresas que abranja empresas de diferentes portes, optou-se por desenvolver uma base que possa representar de forma mais adequada a população de empresas brasileiras. Dessa forma, optou-se por construir uma base abrangendo: (1) cadastro de respondentes originados de pesquisas anteriores; (2) cadastro de gerentes de controladoria de uma empresa de consultoria e treinamento; (3) membros de um grupo de rede social focado em controladoria e contabilidade. A escolha de membros de rede social é justificada em função de que estas redes auxiliam na produção e disseminação de conhecimento e inovações relacionadas ao mundo corporativo (MARTINS et al., 2009).

Portanto, apesar de ser construída por conveniência, entende-se que esta amostra reflete com melhor propriedade o universo de gestores que atuam em empresas brasileiras, uma vez que abrange gestores que atuam em médias empresas, as quais não divulgam dados contábeis.

O questionário foi enviado por correspondência eletrônica, por meio de um serviço de disponibilização de questionário de forma eletrônica.

\subsection{Informações descritivas sobre os respondentes}

As tabelas a seguir (Tabela 1 e Tabela 2) fornecem informações descritivas sobre os respondentes, enquanto que a Tabela 3 permite o entendimento do porte das empresas em que tais respondentes atuam. Com base nas informações contidas nessas tabelas, pode-se considerar que os respondentes atendem ao perfil desejado para a realização desta pesquisa, tendo em vista os objetivos propostos.

Tabela 1. Respondentes por Departamento

\begin{tabular}{|l|c|c|}
\hline Departamento & Qtde & Percentagem \\
\hline Centro de Serviços Compartilhados & 2 & $2,5 \%$ \\
\hline Contabilidade & 10 & $12,5 \%$ \\
\hline Controladoria & 50 & $62,5 \%$ \\
\hline Custos e Orçamentos & 6 & $7,5 \%$ \\
\hline Financeiro & 12 & $15,0 \%$ \\
\hline Total & $\mathbf{8 0}$ & $\mathbf{1 0 0 , 0} \%$ \\
\hline
\end{tabular}

Tabela 2. Respondentes por cargo

\begin{tabular}{c|c|c}
\hline Cargo & Quantidade & Percentagem \\
\hline Chefe & 1 & $1,3 \%$ \\
\hline Coordenador & 15 & $18,8 \%$ \\
\hline Diretor & 12 & $15,0 \%$ \\
\hline Gerente & 41 & $51,3 \%$ \\
\hline Gerente Geral & 6 & $7,5 \%$ \\
\hline Supervisor & 5 & $6,3 \%$ \\
\hline Total & $\mathbf{8 0}$ & $\mathbf{1 0 0 , 0 \%}$ \\
\hline
\end{tabular}

\subsection{Elaboração do questionário}

\subsubsection{Fontes de obtenção de informação}

Para coletar dados relativos à variável "Fontes de obtenção de informação", a maior parte dos itens foi adaptada do estudo de Mol e Birkinshaw (2009). A seguinte assertiva foi colocada aos respondentes:

Por favor, indique a importância de cada uma das principais fontes de conhecimento ou informação utilizadas para gerar inovações nas técnicas de controle gerencial. O horizonte é os últimos dois anos.

Para mensuração quantitativa foi usada a escala: 1. Não Utilizado, 2. Pouca Importância, 3. Média Importância, 4. Grande Importância. O quadro 2 mostra a codificação das variáveis e a referência bibliográfica.

Tabela 3. Porte das empresas

\begin{tabular}{|c|c|c|c|}
\hline \multicolumn{2}{|c|}{$\begin{array}{l}\text { Intervalo de Classes em } \\
\text { Qtdede Empregados }\end{array}$} & \multirow{2}{*}{$\begin{array}{c}\begin{array}{c}\text { Qtde } \\
\text { de empresas }\end{array} \\
11\end{array}$} & \multirow{2}{*}{$\begin{array}{c}\text { Percentagem } \\
13,75 \% \\
\end{array}$} \\
\hline$[0$ & $100]$ & & \\
\hline$[101$ & $500]$ & 18 & $22,50 \%$ \\
\hline$[501$ & $1.000]$ & 14 & $17,50 \%$ \\
\hline$[1.001$ & $5.000]$ & 24 & $30,00 \%$ \\
\hline$[5.001$ & $10.000]$ & 9 & $11,25 \%$ \\
\hline \multicolumn{2}{|c|}{ [ Acima de 10.000} & 4 & $5,00 \%$ \\
\hline \multicolumn{2}{|l|}{ Total } & 80 & $100 \%$ \\
\hline
\end{tabular}

Quadro 2. Fontes de Informação

\begin{tabular}{|c|c|c|c|}
\hline $\begin{array}{c}\text { Fonte de } \\
\text { informação }\end{array}$ & Variável & $\begin{array}{c}\text { Classificação } \\
\text { segundo Mol \& } \\
\text { Birkinshaw (2009) }\end{array}$ & Referencial \\
\hline $\begin{array}{l}\text { Informações obtidas } \\
\text { junto aos outros } \\
\text { departamentos }\end{array}$ & F1 & Interna & $\begin{array}{c}\text { Adaptada } \\
\text { de Mol e } \\
\text { Birkinshaw } \\
\quad(2009)\end{array}$ \\
\hline $\begin{array}{l}\text { Consultores } \\
\text { externos }\end{array}$ & $\mathrm{F} 2$ & Mercado & $\begin{array}{c}\text { Mol e } \\
\text { Birkinshaw } \\
(2009)\end{array}$ \\
\hline Concorrentes & $\mathrm{F} 3$ & Mercado & $\begin{array}{l}\text { Mol e } \\
\text { Birkinshaw } \\
(2009)\end{array}$ \\
\hline Livros & $\mathrm{F} 4$ & Profissional & $\begin{array}{c}\text { Baseado } \\
\text { em Scapens } \\
\text { (1994) }\end{array}$ \\
\hline $\begin{array}{l}\text { Fornecedores de } \\
\text { Software }\end{array}$ & F5 & Mercado & $\begin{array}{l}\text { Mol e } \\
\text { Birkinshaw } \\
(2009)\end{array}$ \\
\hline $\begin{array}{c}\text { Jornais e Revistas } \\
\text { de Negócios }\end{array}$ & F6 & Profissional & $\begin{array}{c}\text { Mol e } \\
\text { Birkinshaw } \\
(2009)\end{array}$ \\
\hline Artigos Acadêmicos & F7 & Profissional & $\begin{array}{c}\text { Baseado em } \\
\text { Van Helden et } \\
\text { al. }(2010)\end{array}$ \\
\hline $\begin{array}{c}\text { Associações de } \\
\text { classe (empresariais } \\
\text { e profissionais) }\end{array}$ & F8 & Profissional & $\begin{array}{l}\text { Mol e } \\
\text { Birkinshaw } \\
(2009)\end{array}$ \\
\hline Rede de contatos & F9 & Profissional & $\begin{array}{c}\text { Calhou e } \\
\text { Starbuck } \\
(2003)\end{array}$ \\
\hline Seminários e Cursos & F10 & Profissional & $\begin{array}{c}\text { Mol e } \\
\text { Birkinshaw } \\
(2009)\end{array}$ \\
\hline
\end{tabular}




\subsubsection{Tipos de mudança de práticas de controle gerencial}

A variável "Tipos de mudança de práticas de controle gerencial" foi operacionalizada a partir do estudo de Sulaiman e Mitchell (2005). Foram incluídos cinco itens que representam diferentes graus de mudança, além de um sexto item que representa a ausência de mudança. $\mathrm{O}$ primeiro item representa o mais alto grau de inovação em que são adicionadas novas técnicas de controle gerencial ainda não existentes.

Dado que os tipos de mudança de práticas de controle gerencial não são excludentes, os respondentes foram solicitados a distribuir 100 pontos, de acordo com o estudo de Aguiar, Pace e Frezatti (2009). Isso significa que os respondentes deveriam atribuir mais pontos para aquilo que identifica melhor a organização, visando caracterizar os tipos de comportamento relacionados à inovação ocorridos no departamento de controladoria nos últimos dois anos com base na assertiva:

Distribua 100 pontos (mais pontos para aquilo que caracteriza melhor a organização) para caracterizar os tipos de inovação (inovação é definida como a inclusão de novas técnicas de controle gerencial e que não existiam em sua organização) ocorridos no departamento de controladoria nos últimos dois anos. Mais de uma resposta é possível.

Não se aplica às inovações introduzidas para

adequação à lei 11.638 e ou IFRS. O quadro 3 apresenta as variáveis relativas a esse construto.

Quadro 3: Tipos de mudança de práticas de controle gerencial

\begin{tabular}{|c|c|c|c|}
\hline Assertiva & $\begin{array}{l}\text { Codificação } \\
\text { das variáveis }\end{array}$ & $\begin{array}{l}\text { Tipos de } \\
\text { Inovação }\end{array}$ & Fonte \\
\hline 1. Introdução de novas técnicas de controle gerencial, ainda não existentes na empresa & GI1 & Adição & $\begin{array}{c}\text { Sulaiman e } \\
\text { Mitchell (2005) }\end{array}$ \\
\hline 2. Introdução de novas técnicas, porém, complementares às técnicas atuais & GI2 & Reposição & $\begin{array}{c}\text { Sulaiman e } \\
\text { Mitchell (2005) }\end{array}$ \\
\hline $\begin{array}{l}\text { 3. Mudança na forma de apresentação das informações, quanto à frequência, tipo de } \\
\text { apresentação, etc. }\end{array}$ & GI3 & $\begin{array}{l}\text { Modificação } \\
\text { do Relatório }\end{array}$ & $\begin{array}{c}\text { Sulaiman e } \\
\text { Mitchell (2005) }\end{array}$ \\
\hline $\begin{array}{l}\text { 4. Mudança de critério de uma técnica já existente, por exemplo, custo médio em vez } \\
\text { de PEPS }\end{array}$ & GI4 & $\begin{array}{l}\text { Modificação } \\
\text { Operacional }\end{array}$ & $\begin{array}{c}\text { Sulaiman e } \\
\text { Mitchell (2005) }\end{array}$ \\
\hline 5. Abandono de uma técnica de controle gerencial, sem reposição por outra & GI5 & Redução & $\begin{array}{c}\text { Sulaiman e } \\
\text { Mitchell (2005) }\end{array}$ \\
\hline 6. Nenhuma modificação foi realizada nesse período & GI6 & Estabilidade & Os autores \\
\hline
\end{tabular}

\section{ANÁLISE E DISCUSSÃO DOS RESULTADOS}

\subsection{Fontes de Informação}

Na tabela 4, são apresentadas as estatísticas em relação às variáveis que compõem o construto "Fontes de Informação", para em seguida iniciar a discussão dos resultados.

Tabela 4: Estatística descritiva das importâncias dadas a cada fonte de informação

\begin{tabular}{|c|c|c|c|c|c|}
\hline Fontes de Informação & $\mathrm{N}$ & Mínima & Máxima & Média & Desvio Padrão \\
\hline Informações obtidas junto aos outros departamentos & 80 & 1 & 4 & 3,60 & 0,704 \\
\hline Consultores externos & 80 & 1 & 4 & 2,84 & 0,834 \\
\hline Concorrentes & 80 & 1 & 4 & 2,91 & 0,917 \\
\hline Livros & 80 & 1 & 4 & 2,99 & 0,787 \\
\hline Fornecedores de Software & 80 & 1 & 4 & 2,96 & 0,818 \\
\hline Jornais e Revistas de Negócios & 80 & 1 & 4 & 3,03 & 0,729 \\
\hline Artigos Acadêmicos & 80 & 1 & 4 & 2,81 & 0,797 \\
\hline Associações de classe (empresariais e profissionais) & 80 & 1 & 4 & 2,98 & 0,941 \\
\hline Rede de contatos & 80 & 1 & 4 & 3,31 & 0,756 \\
\hline Seminários e Cursos & 80 & 1 & 4 & 3,15 & 0,731 \\
\hline
\end{tabular}

Nota-se que a fonte de informação interna, isto é, fundamentada na variável "Informações obtidas junto aos outros departamentos", apresentou a maior média. Isso indica que os profissionais de controladoria estão adotando uma postura de socialização do conhecimento, classificada por Nonaka, Konno e Toyama (2001) como coleta de informações, além do processo acumulação de conhecimento tácito (NONAKA, KONNO e TOYAMA, 2001).
Já as variáveis "Consultores Externos", "Rede de Contatos", "Seminários e Cursos", e "Associações de Classe (empresariais e profissionais)" podem ser classificadas no processo denominado coleta de informação externa (NONAKA, KONNO e TOYAMA, 2001), e os resultados mostram que essas variáveis não têm a mesma importância, com especial destaque para consultores externos com uma das médias menores. Isso pode indicar uma menor relevância do papel dos 
consultores como instrumentos de difusão de inovação de práticas de controle gerencial, o que contradiz com o estudo de Seal (2010), que mostrou o papel dos consultores na institucionalização do EVA®. Porém isso pode ter como explicação o fato de cerca de $50 \%$ da amostra do presente estudo ser constituída por empresas com menos de 1.000 empregados, com acesso mais restrito às empresas de consultoria.

Além disso, os resultados podem estar sugerindo que as fontes de informação que decorrem de um processo formal de obtenção como livros e artigos acadêmicos têm um menor uso, o que pode indicar uma preferência maior dos profissionais em obter informações de forma interativa. Outro ponto de alerta é a confirmação do menor uso de artigos acadêmicos como fonte de informação para os profissionais de controladoria, em linha com o que os próprios acadêmicos reconhecem (MALMI; GRANDLUND, 2009, BALDVINSDOTTIR et al., 2010) porém, sem estudos empíricos. Esses dois resultados podem ser indicativos para que professores e pesquisadores em Contabilidade Gerencial devam efetuar modificações no seu modus operandi de transmitir conhecimento.

\subsection{Exploração dos clusters em função das atividades de inovação}

Este tópico explora como se respondeu a questão de pesquisa e para isso empregou-se a técnica de análise de cluster para explorar o padrão da inovação gerencial que rege as empresas da amostra. O método utilizado foi hierárquico, por meio de técnica Ward, medindo a similaridade entre as observações. Esse método foi utilizado, pois a escolha do perfil de mudança não era excludente, com exceção da assertiva 6 que indicava nenhuma modificação.

A definição do número de agrupamentos foi baseada no dendograma e este sugere a formação de quatro agrupamentos, os quais confrontando com seis tipos de inovação demonstrados no Quadro 1 podem ser vistos na Figura 1.

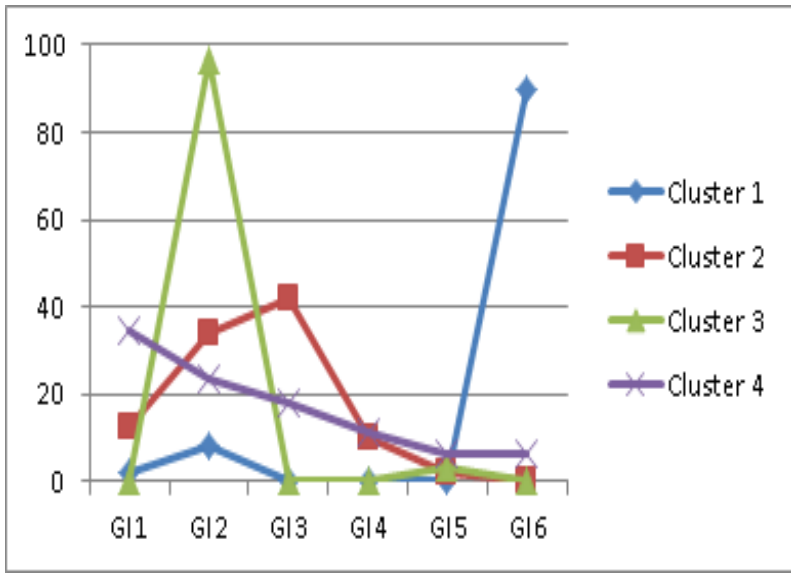

Figura 1. Formação dos clusters por tipos de mudança e fontes de informação

O quadro 4 apresenta os clusters com uma proposta de categorização, considerando a variável predominante de mudança. Para validar os agrupamentos considerando esses indicadores, foi realizado o teste ANOVA das seis variáveis nestes quatro grupos e todos os resultados foram significativamente diferentes $(\mathrm{P}$-value $<0,1)$.
O agrupamento 1 (cluster 1 ) despontou pela alta avaliação para o indicador GI6, comparado com outros grupos, o que demonstra uma postura de inovação caracterizada pela estabilidade das práticas gerenciais, ou seja, com nenhuma modificação durante o período de pesquisa, com apenas cinco casos.

O agrupamento 2 (cluster 2) com 33 casos demonstra que há uma preocupação com os quatros tipos de inovação gerencial (GI1 a GI4), porém, com maior foco destas empresas no GI3, que é a mudança na forma de apresentação das informações, quanto à frequência, ao tipo de apresentação etc. Esses resultados sugerem que essas organizações adotam uma postura de melhoria contínua, na qual a empresa melhora consistentemente fundamentada na aprendizagem de circuito simples (ARGYRIS, 1992), ou seja, pequenas melhorias na forma de evidenciar as informações para os usuários da informação.

Quadro 5. Categorização dos clusters

\begin{tabular}{|c|c|c|c|c|}
\hline$\frac{\dot{ \pm}}{\stackrel{ \pm}{\Xi}}$ & 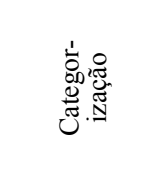 & 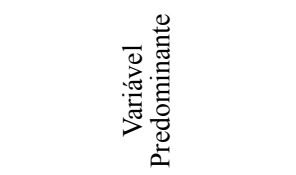 & 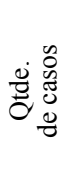 & 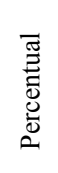 \\
\hline 1 & $\begin{array}{l}\text { Não } \\
\text { Inovadores }\end{array}$ & $\begin{array}{c}\text { GI6 Nenhuma } \\
\text { modificação foi } \\
\text { realizada nesse período }\end{array}$ & 5 & 6,3 \\
\hline 2 & $\begin{array}{l}\text { Inovadores } \\
\text { Contínuos }\end{array}$ & $\begin{array}{l}\text { GI3 Mudança na forma } \\
\text { de apresentação das } \\
\text { informações, quanto } \\
\text { à frequência, tipo de } \\
\text { apresentação, etc.(GI3) }\end{array}$ & 33 & 41,3 \\
\hline 3 & $\begin{array}{l}\text { Inovadores } \\
\text { Incrementais }\end{array}$ & $\begin{array}{l}\text { GI2 Introdução de } \\
\text { novas técnicas, porém, } \\
\text { complementares às } \\
\text { técnicas atuais }\end{array}$ & 3 & 3,8 \\
\hline 4 & $\begin{array}{l}\text { Inovadores } \\
\text { Radicais }\end{array}$ & $\begin{array}{l}\text { GI1 Introdução de novas } \\
\text { técnicas de controle } \\
\text { gerencial, ainda não } \\
\text { existentes na empresa }\end{array}$ & 39 & 48,8 \\
\hline Total & & & 80 & 100 \\
\hline
\end{tabular}

Já o agrupamento 3 (cluster 3) foca na introdução de novas práticas gerenciais complementares às existentes, indicando uma postura de inovação incremental, porém, com apenas três casos.

Por fim, o cluster 4 visa introduzir práticas gerenciais que não existem na empresa, adotando uma postura de inovação radical, e cujo grupo é bastante significativo em termos de quantidade com quase $50 \%$ da amostra.

Os resultados mostram que o tipo de mudança caracterizado como redução é pouco representativo. Dessa forma, pode-se entender que o perfil dos 80 respondentes indica que as empresas adotam uma postura de inovação radical e de melhoria contínua. Para a maior parte das empresas, novas práticas estão sendo introduzidas e, para outra grande parte, as melhorias estão focadas na forma, tipo e frequência de apresentação das informações.

Os resultados são importantes, pois sugerem que há um movimento de inovações de práticas de controle gerencial, práticas estas que não existiam nas organizações até então, uma vez que se somados os dois clusters que representam adoção de inovações e de práticas complementares, chega-se a mais que $50 \%$ da 
amostra. Não é possível afirmar que tipos de inovações estão sendo feitas, mas o que é importante ressaltar é que são técnicas e práticas até então inexistentes nas organizações em que esses profissionais atuam.

Isso de certa forma é um resultado que até então não havia sido mostrado na literatura brasileira, que privilegiou estudos que identificam se determinadas técnicas eram adotadas ou não, porém, com maior foco em grandes empresas. A amostra deste estudo que contempla empresas médias pode também ser um indicativo que, para o universo das médias empresas brasileiras, há sim uma absorção de novos conhecimentos que estão se configurando em novas técnicas de controle gerencial, até então não utilizadas por estas empresas. Esse fenômeno nas médias empresas brasileiras pode ser um indicativo de melhoria de gestão provocado pelo crescimento e perspectivas de entrada de novos investidores no controle dessas organizações.

Já os resultados que mostram uma grande parte das organizações fazendo mudanças em aspectos de comunicação da informação sugerem, ainda que exploratoriamente, que os profissionais de controladoria podem estar focados nos diferentes usuários de informação e, portanto, mais orientados aos negócios (JÄRVENPÄÄ, 2007). Essa postura enseja que o

Tabela 5: Perfil dos tipos de inovação em cada um dos clusters profissional de controladoria deva ter a competência de apresentação de informações, ou seja, além de adequação da linguagem, é necessário desenvolver habilidades de apresentação. Os resultados também sugerem que a dimensão periodicidade do fornecimento de informações tem sido a pauta desses profissionais, o que de certa forma é possibilitado pela existência de sistemas de informações integrados (ERP), já em um estágio em que os profissionais estão envolvidos em geração de relatórios gerenciais por meio de Business Intelligence. Pode-se, ainda depreender que os profissionais de controladoria estão bastante próximos dos usuários, em uma postura de servir aos diferentes usuários das informações.

Dados descritivos referentes ao perfil dos tipos de mudança em cada um dos clusters são apresentados na Tabela 5, tendo como base a classificação por assertiva. Com isso, foi possível perceber em cada cluster, os pesos médios em uma escala de 0 a 100 pontos atribuídos a cada assertiva.

Por exemplo, a Assertiva "Nenhuma modificação foi realizada nesse período" teve uma média no total dos 80 respondentes de 8,8 pontos, mas quando analisado somente o Cluster 1, ela mostra uma média alta de 90 , porém aplicáveis a cinco empresas.

\begin{tabular}{|c|c|c|c|c|c|c|}
\hline Assertiva & Cluster & $\mathbf{N}$ & Média & DP & Mínimo & Máximo \\
\hline \multirow{5}{*}{$\begin{array}{l}\text { Introdução de novas técnicas de controle gerencial, ainda não } \\
\text { existentes na empresa. }\end{array}$} & 1 & 5 & 2 & 4,5 & 0 & 10 \\
\hline & 2 & 33 & 12,7 & 12,9 & 0 & 40 \\
\hline & 3 & 3 & 0 & 0 & 0 & 0 \\
\hline & 4 & 39 & 34,5 & 18,9 & 10 & 100 \\
\hline & Total & 80 & 22,2 & 19,9 & 0 & 100 \\
\hline \multirow{5}{*}{$\begin{array}{l}\text { Introdução de novas técnicas, porém complementares às } \\
\text { técnicas atuais. }\end{array}$} & 1 & 5 & 8 & 11 & 0 & 20 \\
\hline & 2 & 33 & 33,7 & 17,7 & 0 & 70 \\
\hline & 3 & 3 & 96,7 & 5,8 & 90 & 100 \\
\hline & 4 & 39 & 23,3 & 11,2 & 0 & 60 \\
\hline & Total & 80 & 29,4 & 20,5 & $\mathbf{0}$ & 100 \\
\hline \multirow{5}{*}{$\begin{array}{l}\text { Mudança na forma de apresentação das informações, quanto à } \\
\text { frequência, tipo de apresentação, etc. }\end{array}$} & 1 & 5 & 0 & 0 & 0 & 0 \\
\hline & 2 & 33 & 41,6 & 16,6 & 20 & 100 \\
\hline & 3 & 3 & 0 & 0 & 0 & 0 \\
\hline & 4 & 39 & 17,8 & 10,3 & 0 & 40 \\
\hline & Total & 80 & 25,9 & 19,1 & $\mathbf{0}$ & 100 \\
\hline \multirow{5}{*}{$\begin{array}{l}\text { Mudança de critério de uma técnica já existente, por exemplo, } \\
\text { custo médio em vez de peps }\end{array}$} & 1 & 5 & 0 & 0 & 0 & 0 \\
\hline & 2 & 33 & 10,1 & 13 & 0 & 40 \\
\hline & 3 & 3 & 0 & 0 & 0 & 0 \\
\hline & 4 & 39 & 11,2 & 8,7 & 0 & 30 \\
\hline & Total & 80 & 9,7 & 10,7 & $\mathbf{0}$ & 40 \\
\hline \multirow{5}{*}{$\begin{array}{l}\text { Abandono de uma técnica de controle gerencial, sem reposição } \\
\text { por outra }\end{array}$} & 1 & 5 & 0 & 0 & 0 & 0 \\
\hline & 2 & 33 & 1,8 & 5,8 & 0 & 30 \\
\hline & 3 & 3 & 3,3 & 5,8 & 0 & 10 \\
\hline & 4 & 39 & 6,6 & 7,9 & 0 & 30 \\
\hline & Total & 80 & 4,1 & 7,1 & 0 & 30 \\
\hline \multirow{5}{*}{ Nenhuma modificação foi realizada nesse período } & 1 & 5 & 90 & 14,1 & 70 & 100 \\
\hline & 2 & 33 & 0 & 0 & 0 & 0 \\
\hline & 3 & 3 & 0 & 0 & 0 & 0 \\
\hline & 4 & 39 & 6,6 & 10,8 & 0 & 50 \\
\hline & Total & 80 & 8,8 & 22,8 & $\mathbf{0}$ & 100 \\
\hline
\end{tabular}




\subsection{Relação da fonte de conhecimento com as práticas de inovação}

Testes ANOVA foram realizados para verificar se havia diferença entre as fontes de informação e os perfis de mudanças de práticas, cujas estatísticas podem ser visualizadas na Tabela 6 .

Ao realizar esses testes, nota-se que, exceto as fontes de conhecimento F6 (jornais e revistas de negócios), F7 (artigos acadêmicos) e F8 (associações de classe), todas as outras sete tiveram diferença não significativa entre os grupos $(\mathrm{P}$-value $>0,05)$. Diante disso, pode-se depreender que essas fontes de informação não são determinantes para os tipos de inovação, com os resultados sugerindo homogeneidade para estas sete fontes de informação, ou seja, para estas sete fontes de informação não há qualquer diferença em termos dos perfis de mudança, indicando que outros fatores não investigados neste trabalho poderiam explicar as diferenças de perfis de mudança de práticas de controle gerencial. Dentre esses fatores, pode ser destacado o estágio pelo qual a organização está passando em termos de profissionalização da gestão, inovações organizacionais, perspectivas estratégicas, desempenho etc.

Em contrapartida, o resultado da ANOVA indica que existem diferenças entre os quatro clusters quanto aos pesos atribuídos às fontes $\mathrm{F} 6(\mathrm{P}$-value $=0,04), \mathrm{F} 8$ $(\mathrm{P}$-value $=0,03)$ e $\mathrm{F} 7$, apesar de esse último ser pouco significativo $(\mathrm{P}-\mathrm{value}=0,07)$, porém aceitável em função de ser um estudo exploratório. Isso ocorre somente para o cluster 1, que é caracterizado como Não Inovadores. Esse grupo atribuiu maior importância à fonte F6 (jornais e revistas de negócio), quando comparado aos outros grupos (média $=3,8$, P-value $=0,037$ ), além de ter atribuído mais atenção à fonte $\mathrm{F} 8$ (associações de classe) (média cluster $1=3,8, \mathrm{P}$-value $<0,1)$ e aos artigos acadêmicos (média $=3,6, \mathrm{P}$-value $=0,07$ ).

Tabela 6. Testes ANOVA

\begin{tabular}{|c|c|c|c|c|c|c|}
\hline & & SQ & Gl & MQ & $\mathbf{F}$ & Sig. \\
\hline \multirow{3}{*}{$\begin{array}{l}\text { F1 - Informações obtidas junto aos outros } \\
\text { departamentos }\end{array}$} & Entre grupos & 2,322 & 3 & 0,774 & 1,595 & 0,198 \\
\hline & Dentre grupos & 36,878 & 76 & 0,485 & & \\
\hline & Total & 39,2 & 79 & & & \\
\hline \multirow{3}{*}{ F2 - Consultores externos } & Entre grupos & 1,128 & 3 & 0,376 & 0,532 & 0,662 \\
\hline & Dentre grupos & 53,759 & 76 & 0,707 & & \\
\hline & Total & 54,887 & 79 & & & \\
\hline \multirow{3}{*}{ F3 - Concorrentes } & Entre grupos & 0,308 & 3 & 0,103 & 0,118 & 0,949 \\
\hline & Dentre grupos & 66,079 & 76 & 0,869 & & \\
\hline & Total & 66,388 & 79 & & & \\
\hline \multirow{3}{*}{ F4 - Livros } & Entre grupos & 3,334 & 3 & 1,111 & 1,85 & 0,145 \\
\hline & Dentre grupos & 45,653 & 76 & 0,601 & & \\
\hline & Total & 48,988 & 79 & & & \\
\hline \multirow{3}{*}{ F5 - Fornecedores de Software } & Entre grupos & 1,319 & 3 & 0,44 & 0,648 & 0,587 \\
\hline & Dentre grupos & 51,568 & 76 & 0,679 & & \\
\hline & Total & 52,888 & 79 & & & \\
\hline \multirow{3}{*}{ F6 - Jornais e Revistas de Negócios } & Entre grupos & 4,246 & 3 & 1,415 & 2,853 & $0,043 *$ \\
\hline & Dentre grupos & 37,704 & 76 & 0,496 & & \\
\hline & Total & 41,95 & 79 & & & \\
\hline \multirow{3}{*}{ F7 - Artigos Acadêmicos } & Entre grupos & 4,498 & 3 & 1,499 & 2,494 & $0,066^{*}$ \\
\hline & Dentre grupos & 45,69 & 76 & 0,601 & & \\
\hline & Total & 50,187 & 79 & & & \\
\hline \multirow{3}{*}{$\begin{array}{l}\text { F8 - Associações de classe (empresariais e } \\
\text { profissionais) }\end{array}$} & Entre grupos & 7,891 & 3 & 2,63 & 3,221 & $\mathbf{0 , 0 2 7 *}$ \\
\hline & Dentre grupos & 62,059 & 76 & 0,817 & & \\
\hline & Total & 69,95 & 79 & & & \\
\hline \multirow{3}{*}{ F9 - Rede de contatos } & Entre grupos & 2,216 & 3 & 0,739 & 1,306 & 0,279 \\
\hline & Dentre grupos & 42,972 & 76 & 0,565 & & \\
\hline & Total & 45,187 & 79 & & & \\
\hline \multirow{3}{*}{ F10 - Seminários e Cursos } & Entre grupos & 0,132 & 3 & 0,044 & 0,079 & 0,971 \\
\hline & Dentre grupos & 42,068 & 76 & 0,554 & & \\
\hline & Total & 42,2 & 79 & & & \\
\hline
\end{tabular}


Os resultados sugerem que o cluster 1 se destaca dos outros clusters em relação a essas fontes de informação, e o curioso é que esse agrupamento corresponde ao grupo que não realizou modificação alguma durante o período pesquisado, muito embora com apenas cinco casos. Algumas possíveis explicações, sujeitas às futuras pesquisas podem ser elaboradas:

- Isso pode indicar que essas organizações estão buscando nessas fontes de informação o conhecimento necessário para avaliar a real necessidade de mudanças das práticas de controle gerencial.

- Esse grupo pode ser mais crítico em relação às inovações, adotando uma postura de analisálas criticamente, em um cenário no qual as novas técnicas de controle gerencial sejam caracterizadas como não solucionadoras dos problemas de controle gerencial. Essa postura crítica pode ser influenciada pela fonte de informação F7 (Artigos acadêmicos, com média igual a 3,6 e P-value igual a 0,07), uma vez que profissionais que focam em conhecimentos acadêmicos podem também apresentar um nível de postura crítica mais elevada, o que pode levá-los a elaborar uma análise mais aprofundada dos benefícios de novas práticas para as organizações, já que muitas das práticas gerenciais podem não se manifestar em resultado da forma como se espera (HENDRICKS et al., 2007; HULT et al., 2007; POWELL, 1995) e que nem sempre estas garantem o sucesso no longo prazo (WOOD JR; PAULA, 2002).

- Já em relação às duas outras fontes de informação, os resultados podem indicar que profissionais também podem adquirir postura mais crítica por meio de reuniões em associações de classe e associações empresariais, onde comumente há uma discussão mais aprofundada dos benefícios de novas práticas, no que pode ser caracterizado como uma comunidade de práticos. Em relação aos jornais e revistas de negócio, estes evoluíram consideravelmente nos últimos tempos, adotando uma postura mais investigativa quando divulgam informações sobre as práticas de controle gerencial adotadas pelas empresas, o que pode contribuir para essa postura crítica.

- Outra explicação provável é que as organizações alocadas nesse cluster já estejam em um estágio em que as inovações já tenham sido realizadas.

Para elucidar essas explicações são apresentadas as tabelas 7 a 9, nas quais são destacadas as Fontes F6, F7 e F8.

Tabela 7: Média das importâncias atribuídas pelos 4 clusters para fonte F6 Jornais e Revistas de Negócios

\begin{tabular}{c|c|c|c|c|c|c}
\hline Cluster & Denominação & $\mathbf{N}$ & Média & DP & Mínimo & Máximo \\
\hline 1 & Não Inovadores & 5 & 3,80 & 0,45 & 3,00 & 4,00 \\
2 & Inovadores Contínuos & 33 & 3,06 & 0,75 & 1,00 & 4,00 \\
3 & Inovadores Incrementais & 3 & 3,33 & 0,58 & 3,00 & 4,00 \\
4 & Inovadores Radicais & 39 & 2,87 & 0,70 & 2,00 & 4,00 \\
\hline Total & & $\mathbf{8 0}$ & $\mathbf{3 , 0 3}$ & $\mathbf{0 , 7 3}$ & $\mathbf{1 , 0 0}$ & $\mathbf{4 , 0 0}$ \\
\hline
\end{tabular}

Tabela 8: Média das importâncias atribuídas pelos 4 clusters para fonte F8 Associações de Classes (empresariais e profissionais)

\begin{tabular}{c|c|c|c|c|c|c}
\hline Cluster & Denominação & N & Média & DP & Mínimo & Máximo \\
\hline 1 & Não Inovadores & 5 & 3,80 & 0,45 & 3,00 & 4,00 \\
2 & Inovadores Contínuos & 33 & 3,12 & 0,89 & 1,00 & 4,00 \\
3 & Inovadores Incrementais & 3 & 2,00 & 1,00 & 1,00 & 3,00 \\
4 & Inovadores Radicais & 39 & 2,82 & 0,94 & 1,00 & 4,00 \\
\hline Total & & $\mathbf{8 0}$ & 2,96 & $\mathbf{0 , 9 4}$ & $\mathbf{1 , 0 0}$ & $\mathbf{4 , 0 0}$ \\
\hline
\end{tabular}

Tabela 9: Média das importâncias atribuídas pelos 4 clusters para fonte F7 Artigos Acadêmicos

\begin{tabular}{l|c|c|c|c|c|c}
\hline Cluster & Denominação & N & Média & DP & Mínimo & Máximo \\
\hline 1 & Não Inovadores & 5 & 3,60 & 0,55 & 3,00 & 4,00 \\
2 & Inovadores Contínuos & 33 & 2,88 & 0,78 & 1,00 \\
3 & Inovadores Incrementais & 3 & 3,00 & 1,00 & 2,00 \\
4 & Inovadores Radicais & 39 & 2,64 & 0,78 & 1,00 & 4,00 \\
\hline Total & & $\mathbf{8 0}$ & $\mathbf{2 , 8 1}$ & $\mathbf{0 , 8 0}$ & $\mathbf{1 , 0 0}$ & $\mathbf{4 , 0 0}$ \\
\hline
\end{tabular}

Para auxiliar no entendimento dos demais clusters é apresentada a Tabela 10, a qual destaca as notas médias que variavam entre 1 a 4.

Tabela 10: Média das importâncias atribuídas pelos 4 clusters todas as fontes

\begin{tabular}{|c|c|c|c|c|c|c|c|c|c|c|c|}
\hline Cluster & Denominação & F1 & F2 & F3 & F4 & F5 & F6 & F7 & F8 & F9 & F10 \\
\hline 1 & Não Inovadores & 3,40 & 2,60 & 3,00 & 3,20 & 3,40 & 3,80 & 3,60 & 3,80 & 3,40 & 3,20 \\
\hline 2 & Inovadores Contínuos & 3,52 & 2,97 & 2,88 & 3,06 & 2,91 & 3,06 & 2,88 & 3,12 & 3,45 & 3,18 \\
\hline 3 & Inovadores Incrementais & 3,00 & 2,67 & 2,67 & 2,00 & 2,67 & 3,33 & 3,00 & 2,00 & 2,67 & 3,00 \\
\hline 4 & Inovadores Radicais & 3,74 & 2,77 & 2,95 & 2,97 & 2,97 & 2,87 & 2,64 & 2,82 & 3,23 & 3,13 \\
\hline
\end{tabular}


Sobre o cluster 4, que tem maior número de casos, nota-se que em relação às fontes F6 (Jornais e Revistas de Negócios)e F8 (Artigos Acadêmicos), ele é o agrupamento com as menores médias. Esses resultados podem indicar um grupo menos influenciado por aspectos de conhecimento explícito.

Enquanto o cluster 2, com o segundo maior número de casos, tem as maiores médias para as fontes F1 (informações de outros deptos) e F9 (rede de contatos), isso pode indicar um grupo que aprende de forma colaborativa, tanto com fontes internas como de externas.

\section{CONSIDERAÇ̃̃ES FINAIS}

O presente trabalho teve como objetivo avaliar como os profissionais de controladoria adquirem informações e as transformam em mudanças de práticas de controle gerencial. Este estudo possui grande importância, uma vez que a literatura aponta para um quadro de estabilidade das práticas de controle gerencial, e um estudo que associasse estas duas variáveis ainda não existia.

Por meio de análise de clusters, foram identificados quatro agrupamentos denominados de "Não Inovadores", "Inovadores Frequentes", "Inovadores Incrementais" e "Inovadores Radicais", e os principais achados deste estudo demonstraram que a fonte interna de informação é considerada como a mais importante para a obtenção de conhecimento, seguida por rede de contatos e seminários e cursos. A hipótese que declarava que haveria diferenças entre a forma como se obtém informação e perfis de mudanças de controle gerencial não foi comprovada, indicando que outros fatores podem explicar os diferentes perfis de mudanças de práticas de controle gerencial. A única exceção se refere ao agrupamento denominado "Não Inovadores", em que as fontes de informação como jornais e revistas de negócios, artigos acadêmicos e associações de classe são diferentes em relação aos demais perfis, com médias maiores, o que pode indicar uma postura crítica em relação às inovações ou mesmo que estas organizações ainda estão buscando informações para um futuro movimento de mudanças.

As limitações da pesquisa se referem à amostra ter sido obtida por conveniência, além de estes resultados estarem baseados em percepções, as quais podem ter viés nas respostas.

Estudos futuros poderiam identificar outros fatores que poderiam influenciar os diferentes perfis de mudanças de controle gerencial, como a questão de recursos, tamanho e complexidade, na perspectiva contingencial; e comportamentos miméticos suportados pela Teoria Institucional. Estudos de caso longitudinais em organizações classificadas nos diferentes clusters poderiam trazer contribuições mais aprofundadas sobre esses fenômenos.

\section{REFERÊNCIAS}

AGUIAR, A.B.; GUERREIRO, R. Processos de persistência e mudança de sistemas de contabilidade gerencial: uma análise sob o paradigma institucional. Revista Universo Contábil, v. 4, n. 3, p. 6-24,
2008.

AGUIAR, A. B. ; PACE, E. S. U. ; FREZATTI, F . Análise do inter-relacionamento das dimensões da estrutura de sistemas de controle gerencial: um estudo piloto. RAC Eletrônica (Online), v. 3, p. 1-21, 2009.

ARGYRIS, C. Enfrentando defesas empresariais. Rio de Janeiro, Campus, 1992.

ALMEIDA, P.; PHENE, A.; GRANT, R. Innovation and Knowledge Management: Scanning, Sourcing and Integration. In: EASTERBY-SMITH, M.; LYLES, M.A. (eds.) The Blackwell handbook of organizational learning and knowledge management. Blackwell, 2003

BALDVINSDOTTIR, G., MITCHELL, F. NøRREKLIT, $H$. Issues in the relationship between theory and practice in management accounting. Management Accounting Research, v. 21, n. 2, p.79-82, 2010.

BERRY, A.J.; COAD, A.F.; HARRIS, E.P.; OTLEY, D.T.; STRINGER, C. Emerging themes in management control: A review of recent literature. The British Accounting Review, v.41, n. 1, p. 2-20, 2009.

CALHOU, M.A.; STARBUCK, W.H. Barriers to Creating Knowledge. In: EASTERBY-SMITH, M.; LYLES, M.A. (eds.) The Blackwell handbook of organizational learning and knowledge management. Blackwell, 2003.

CHEN, C.; HUANG, J. Strategic human resource practices and innovation performance - The mediating role of knowledge management capacity. Journal of Business Research, v. 62, n. 1, p. 104114, 2009.

CHENHALL, R. H. Integrative strategic performance measurement systems, strategic alignment of manufacturing, learning and strategic outcomes: an exploratory study. Accounting. Organizations and Society, v. 30, n. 5, p. 395-422, 2005.

CHENHALL, R. H. Accounting for the horizontal organization: A review essay. Accounting, Organizations and Society, v. 33, n. 4-5, p. 517$550,2008$.

CHO, Chun Wei. The knowing organization: how organizations use information to construct meaning, create knowledge, and make decisions. Oxford, $2^{\text {nd }}$ edition, 354 p., 2006.

DAVILA, A.; FOSTER, G.; OYON, D. Accounting and Control, Entrepreneurship and Innovation: Venturing into New Research Opportunities. European Accounting Review, v. 18, n. 2, p. 281311,2009

DILLMAN, D.A. Mail and Internet Surveys: the tailored design model. Wiley, 2000.

EMSLEY, D. Restructuring the management accounting function: A note on the effect of role involvement on innovativeness. Management Accounting 
Research, v. 16, n. 2, p. 157-177, 2005.

FLEURY, A.; FLEURY, M. T. L. Estratégias empresariais e formação de competências: um quebra-cabeça caleidoscópico da indústria brasileira. S. Paulo: Atlas, 2000.

FLYCK, Uwe. Introdução à Pesquisa Qualitativa. $3^{a}$ ed. Porto Alegre: Bookman, 2009.

GRANT, R. M. Toward knowledge-based theory of the firm. Strategic Management Journal, v. 17, special issue, p. 109-122, 1996.

HAIR JR., J. F.; ANDERSON, R.E.; BLACK, W. Análise multivariada de dados. Porto Alegre: Bookman, 2005.

HENDRICKS, K. B.; SINGHAL, V. R.; STRATMAN, J. K. The impact of enterprise systems on corporate performance: A study of ERP, SCM, and CRM system implementations. Journal of Operations Management, v. 25, n. 1, p. 65-82, 2007.

HULT, G. T. M.; KETCHEN, D. J.; ARRFELT, M. Strategic supply chain management: Improving performance through a culture of competitiveness and knowledge development. Strategic Management Journal, v. 28, n. 10, p. 1035-1052, 2007.

JÄRVENPÄÄ, M. Making Business Partners: A Case Study on how Management Accounting Culture was Changed. European Accounting Review, v. 16, n. 1, p. 99-142, 2007.

MALMI, T.; GRANLUND, M. In search of Management Accounting Theory. European Accounting Review, v. 18, n. 3, p. 597-620, 2009.

MARTINS, J.T.; QUINCOZES, E.R.F.; PEREIRA, M.F; FIALHO, F.A.P. A contribuição das redes sociais para o desenvolvimento de projetos de pesquisa, desenvolvimento e inovação (P,D\&I): o caso da Embrapa. Simpósio de Excelência em Gestão e Tecnologia (2009). Disponível em WWW.aedb.br/ seget/artigos09/290_artigo.pdf. Acesso em 01 de setembro de 2011.

MOL, M.J.; BIRKINSHAW, J. The sources of management innovation: When firms introduce new management practices. Journal of Business Research, v. 62, n. 12, p.1269-1280, 2009.

NONAKA, I.; KONNO,N.; TOYAMA, R. Emergence of "Ba": A Conceptual Framework for the continuous and Self-transcending Process of Knowledge Creation.p.13-29. In: NONAKA, I.; NISHIGUCHI, T. (eds) Knowledge Emerge: Social, Technical, and Evolutionary Dimensions of Knowledge Creation. 303p. Oxford, 2001.

PETTIGREW, A.M.; MASSINI, S. Innovative forms of organizing: trends in Europe, Japan and the USA in the 1990s. Sage Publications, 2003.

POWELL, T. C. Total quality management as competitive advantage: A review and empirical study. Strategic
Management Journal, v. 16, n. 1, p. 15-37, 1995.

SCAPENS, R.W. Never mind the gap: towards an institutional perspective on management accounting practice. Management Accounting Research, v. 5, n. 3/4, p. 301-321, 1994.

SEAL, W. Managerial discourse and the link between theory and practice: from ROI to value-based management. Management Accounting Research, v.21, p.95-109, 2010.

STONE, D.N.; HUNTON, J.E.; WIER, B. Succeeding in managerial accounting. Part 1: knowledge, ability, and rank. Accounting, Organizations and Society, v. 25, n. 7 , p. $697-715,2000$.

SULAIMAN, S.; MITCHELL, F. Utilizing a typology of management accounting change: An empirical analysis. Management Accounting Research, v. 16, n. 4, p. 422-437, 2005.

SUNDBO, J. The Strategic Management of Innovation: a sociological and economic theory. Edward Elgar, 2001.

VAN HELDEN, G. J.; AARDEMA, H.; TER BOGT, H.J., GROOT, T.L.C.M. Knowledge creation for practice in public sector management accounting by consultants and academics: Preliminary findings and directions for future research. Management Accounting Research, v. 21, n. 2, p. 83-94, 2010.

VASCONCELOS, F.C. Dinâmica Organizacional e Estratégia: Imagens e Conceitos. São Paulo: Pioneira Thomson Learning, 2007.

WOOD JR, T.; PAULA, A. P. P. Pop-management: contos de paixão, lucro e poder. Organizações e Sociedade, v. 9, n. 24, p. 39-51, 2002. 